\title{
Experimental and Numerical Design and Optimization of a Counter-Flow Heat Exchanger
}

\author{
Salman Bahrami ${ }^{1}$ and Mohammad Hassan Rahimian ${ }^{2}$ \\ ${ }^{1}$ Mechanical Engineering Department, University of Tehran, Tehran, Iran \\ ${ }^{2}$ Mechanical Engineering Department, University of Tehran, Tehran, Iran
}

\begin{abstract}
A new inexpensive counter-flow heat exchanger has been designed and optimized for a vapor-compression cooling system in this research. The main aim is to experimentally and numerically evaluate the effect of an internal heat exchanger (IHX) adaptation in an automotive air conditioning system. In this new design of IHX, the highpressure liquid passes through the central channel and the low-pressure vapor flows in several parallel channels in the opposite direction. The experimental set-up has been made up of original components of the air conditioning system of a medium sedan car, specially designed and built to analyze vehicle $\mathrm{A} / \mathrm{C}$ equipment under real operating conditions. The results show that this compact IHX may achieve up to $10 \%$ of the evaporator capacity while low pressure drop will be imposed on this refrigeration cycle. Also, they confirm considerable decrease of compressor power consumption (CPC), which is intensified at higher evaporator air flow. A significant improvement of the coefficient of performance (COP) is achieved with the IHX employment too. The influence of operating conditions has been also discussed in this paper. Finally, numerical analyses have been briefly presented, which bring more details of the flow behavior and heat transfer phenomena, and help to determine the optimal arrangement of channels.
\end{abstract}

\section{Introduction}

In cooling/refrigeration industry, liquid-suction or internal heat exchangers (IHXs) are installed in vaporcompression refrigeration systems to exchange energy between relatively cool low-pressure gas and warm highpressure liquid refrigerants. The main advantages of the IHX installation are: increasing refrigerating effect at the evaporator, sub-cooling liquid refrigerant to ensure liquid phase entrance to the expansion valve, and minimizing the risk of liquid refrigerant entering at the compressor inlet. The possible disadvantages can be: decreasing the refrigerant mass flow rate delivered by the compressor, increasing compressor discharge temperature, and increasing pressure losses at the suction and liquid lines $[1,2]$.

Using IHX has been studied mostly in $\mathrm{CO} 2$ refrigeration systems. It is an important factor to achieve high system performance; therefore some research works focusing on $\mathrm{CO} 2$ trans-critical systems with IHX have been reported [3-8].

Mastrullo at el. [9] presented a chart for predicting the effectiveness of IHX installation for 19 different ozone friendly fluids, and concluded that the advisability of the IHX usage can be evaluated as a function of thermodynamic properties. Variations of evaporating and condensation temperatures were respectively in the range of $-40{ }^{\circ} \mathrm{C} / 10{ }^{\circ} \mathrm{C}$ and $25{ }^{\circ} \mathrm{C} / 50{ }^{\circ} \mathrm{C}$. Klein at el. [10] identified a dimensionless group to correlate performance impacts attributable to the IHXs, and studied the impact of pressure drops through the IHXs on system performance. They concluded that for given operating conditions the advantages are higher for R134a than $\mathrm{R} 407 \mathrm{C}$ and R22.

\section{IHX design for a cooling system}

In the present work, the effects of adopting a new IHX in an R134a automotive A/C system have been analyzed. The evaporator calorimeter tests have been carried out to evaluate the influences of adaptation of the designed IHX on the cycle performance parameters, which are quite important from point of views of system performance optimization, power saving and fuel consumption.

The IHX is of the counter-flow type made up of aluminum extruded channels. In this design, the relatively warm liquid refrigerant, which is leaving the condenser, passes through the central channel. The relatively cold low-pressure vapor, which is exiting the evaporator, flows in several parallel channels in the opposite direction. The inner- and outer diameters of the IHX have been determined based on geometries of inlet and outlet pipes of the refrigeration system connected to the IHX. The thickness of walls has been mainly dictated by manufacturing constraints. Several IHX lengths have been employed and evaluated from $10 \mathrm{~cm}$ up to $30 \mathrm{~cm}$.

The manufacturing process of this type of IHX is simpler and less expensive in comparison with most other 
IHXs. In addition, it is light and compact; so quite useful in the limited space of automobiles. Although it has been designed for automotive $\mathrm{A} / \mathrm{C}$ systems, but the same concept can be implemented in other cooling systems in different industries such as aerospace and oil \& gas.

\section{Experimental investigations}

\subsection{Experimental set-up}

The experimental set-up has been made up of original components from the air conditioning system of a medium sedan car, arranged in such a way to emulate them in the actual vehicle. It has been made up of three fluid circuits; the refrigerant, the evaporator air, and the condenser air. The diagram of the test apparatus can be found in Ref. [2]. Fig. 1 shows the condenser room with the metal frame in front of the condenser, where 12 thermocouples have been installed on it to find the average temperature of the air flow [2].

$\mathrm{R} 134 \mathrm{a}$ is used in experimental test bench as the refrigerant. Temperature and pressure of refrigerant are measured with the $\mathrm{T}$ type thermocouples and pressure transmitters, respectively. The mass flow rate of refrigerant is measured with a Coriolis type meter with the capacity of $0-225 \mathrm{~kg} / \mathrm{h}$ with $1 \mathrm{~kg} / \mathrm{h}$ precision, installed in the liquid line. Input parameters of evaporator calorimeter test of automobile $\mathrm{A} / \mathrm{C}$ system are evaporator air flow rate, dry bulb and wet bulb temperatures of evaporator inlet air, expansion valve inlet pressure, and evaporator average pressure. The condenser is a parallel flow type containing 34 multi-channel flat tubes, which is used in this type of car. The installed evaporator on the system is a laminated type. The evaporator air chamber has been set in the dry bulb temperature of $27^{\circ} \mathrm{C}$ and $50 \%$ relative humidity. Sensible and latent thermal loads of evaporator have been provided by a heater and a humidifier installed upstream of the evaporator. The evaporator blower passes the air flow rate of 200, 300, 400 and $500 \mathrm{~m}^{3} / \mathrm{h}$. Average pressure of evaporator has been set in $0.25 \mathrm{MPa}$ gage. Two inlet expansion valve pressures have been considered; 20 and 23 bar gage. The compressor is a reciprocating swash plate one with the capacity of $150 \mathrm{cc} / \mathrm{rev}$.

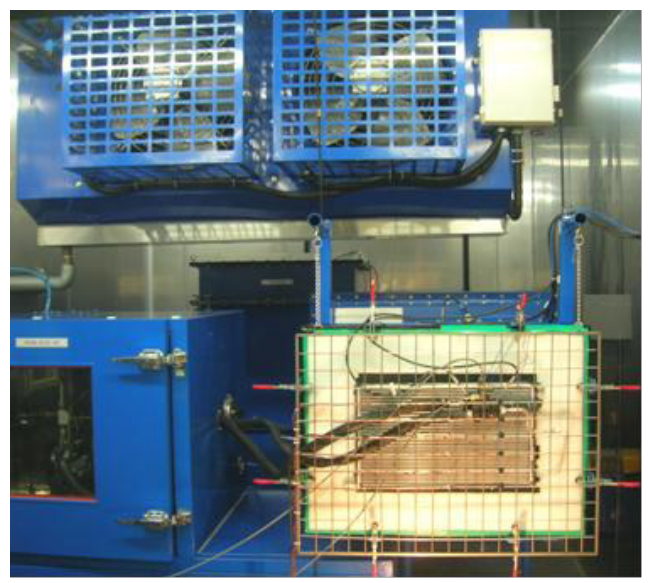

Figure 1. Condenser test room.

\subsection{Experimental results}

The results indicate that pressure drops of the highpressure line are lower than the low-pressure line; mainly since the low-pressure flow passes through several parallel channels in contacts with more walls, which causes more friction losses. The pressure drops of the low-pressure vapor over passing through two IHXs of 10 and $30 \mathrm{~cm}$ are respectively equal to $2.9 \%$ and $6.1 \%$ of the inlet pressure, which are suitable in comparison with other IHXs. The capacity of IHX is equal to multiplication of refrigerant mass flow rate and difference of inlet and outlet enthalpies, which could be calculated from temperature and pressure of the inlet- and outlet refrigerant. The highest capacity of IHXs belongs to the higher pressure of expansion valve inlet. It is noticeable that in $30 \mathrm{~cm} \mathrm{IHX,} \mathrm{increasing} \mathrm{evaporator} \mathrm{air}$ flow causes enhancement of IHX capacity. The results show that by IHX length increase from $10 \mathrm{~cm}$ to $30 \mathrm{~cm}$, its capacity will improve $690 \%$ and $280 \%$ in expansion valve inlet pressure of 20 bar gage and 23 bar gage respectively. IHX capacity is usually compared with the capacity of other components. For instant, Fig. 2 shows the IHX/evaporator capacity ratio obtained in different evaporator air flows. In the best test conditions, the 30 cm IHX capacity is $13 \%$ of fabric evaporator capacity.

The most significant effects of IHX employment is related to the compressor performance. Using IHX leads to a maximum CPC reduction of $25 \%$. Installation of 10 $\mathrm{cm}$ and $30 \mathrm{~cm}$ IHXs causes respectively $13 \%$ and $16 \%$ $\mathrm{CPC}$ reduction in average. The aforementioned achievements can lead to significant automobile net engine power enhancement, as well as fuel economy improvement. Those $\mathrm{CPC}$ reductions result the cycle COP increase by $5.4 \%$ and $7.7 \%$ in average respectively.

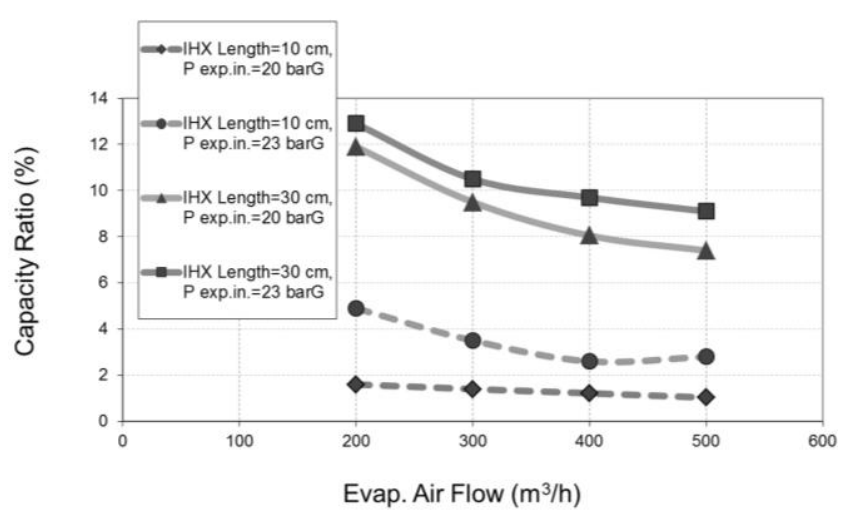

Figure 2. Capacity ratio versus evaporator air flow.

\section{Numerical analyses}

First, structural analyses were carried out to ensure proper arrangement of fluids in central and outer channels from the maximum stress perspective. Then CFD analyses were executed to learn more about the fluidic/thermal phenomena happening in the IHX. Also several numbers of outer parallel channels were investigated. 


\subsection{Structural analyses}

Static finite element analyses were carried out for two possible configurations of the liquid- and vapor counter flows. Fig. 3 shows the results for the appropriate configuration that poses a lower maximum stress. In this configuration the stress concentration is around the internal channel that contains the high-pressure liquid. The maximum von-Mises stress $(6.9 \mathrm{MPa})$ indicates a proper safe margin, about $6 \%$, considering the properties of the specific Aluminum alloy used. The other configuration (i.e. vapor flows in the central channel) may impose about a 5-times higher maximum stress into the component.
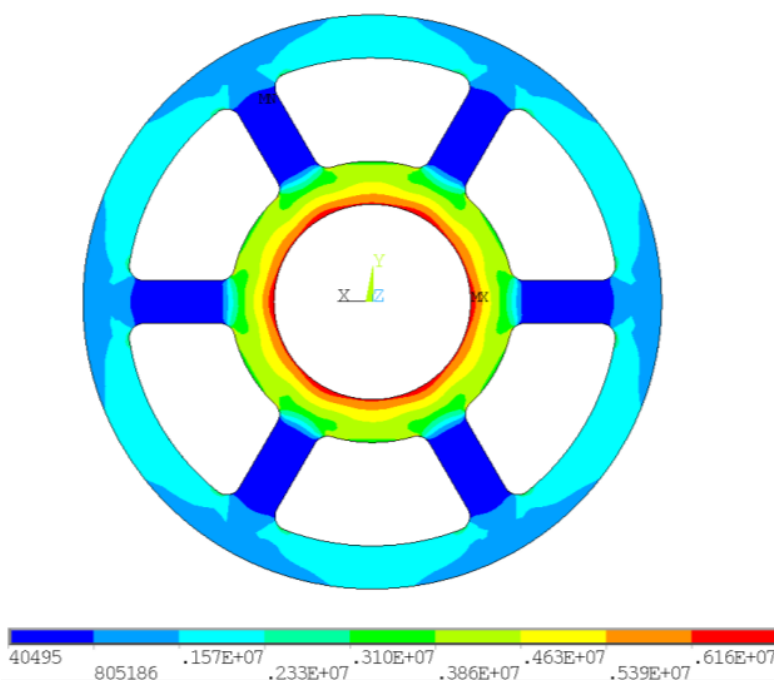

Figure 3. Stress contours (in Pa).

\subsection{CFD analyses}

Ansys CFX software was used to solve the equations in three different computational domains (i.e. vapor, liquid, and solid), and to obtain velocity, pressure, and temperature contours through them. Steady state condition and second order upwind approach were applied for momentum and energy equations. Boundary conditions were defined according to the experiments, and the results were validated at the same operating conditions. Mass flow inlet boundary conditions for both fluids indicate that flows are quite turbulent; so a k- $\varepsilon$ turbulent model was employed according to previous researches and the computational budget. Due to similarity of parallel channels only one channel was simulated with the proportional slice of the central channel, using rotational periodic boundary condition. Proper initializations were considered to obtain the convergence. Mesh independency was studied as well.

The results indicate that pressure drops are wellconsistent with experimental investigations, especially for the low-pressure vapor. Heat transfer parameters are also well predicted according to comparison of average outlet temperatures for both fluids. For instance, Fig. 4 shows the temperature contours in the cross section of the IHX with six parallel channels. The big temperature difference between the cores of two fluids (i.e. $63^{\circ} \mathrm{C}$ ) is obvious in this figure, which produces big temperature gradients through the IHX. In addition, producing relatively hot boundary layers inside the outer channels illustrates the proper heat transfer mechanism through the hot walls into the cold channels. In this figure the relatively cold vapor leaves the IHX towards the compressor. The average superheat degree of refrigerant is $7.4^{\circ} \mathrm{C}$, which is quite good from the compressor performance perspective. Fig. 5 shows surface heat transfer coefficient contours along the central channel for the aforementioned IHX. These contours are similar to heat flux contours. The parts of walls close to the liquid entrance have lower heat fluxes due to transferring the heat with the vapor that is much warmer when it is leaving the IHX. The results indicate that heat transfer coefficients are less than $50 \%$ in outer channels.

The number of parallel channels was investigated numerically. All walls thicknesses and inner- and outer diameters remained constant. From the structural perspective, there is no significant change in the stress contours. From the fluidic point of view, increasing the number of channels reduces the inlet flow area, so in a constant mass flow rate the vapor would enter with a higher velocity. Although it brings a higher convection coefficient, but increases pressure drops as well. While the velocity and heat transfer area are unchanged in the liquid side, increasing the number of parallel channels expands the heat transfer surfaces in the vapor side, and consequently increases the heat absorption potential. It should be noted that the Reynold's number of liquid flow is about up to three times higher than the vapor one. Considering all the aforementioned points and trade-off, based on the numerical results the number of five channels has been identified as the optimum number of channels for the current application.

\section{Conclusion and future works}

The experimental- and numerical results show that using this proposed IHX can bring significant positive impacts on the performance of car refrigeration cycles. Also, using the studied heat exchanger would impose low pressure drops on the cycle.

Improvement of the $\mathrm{CPC}$ is one of the most important achievements of IHX installation. It can increase automobile net engine power, improve its efficiency, and bring economy of fuel. Significant COP enhancement would be expected in the refrigeration cycle as well.

As future works, different mechanisms will be considered to enhance the IHX heat transfer efficiency. For instance adding inner and outer grooves and fines will be studied in order to increase heat transfer surfaces and thermal coefficients. All geometric parameters can be optimized in the next step in order to improve IHX capacity while pressure drops do not increase significantly. Finally, using other refrigerants in other cooling applications will be studied, such as R407C and R22. 

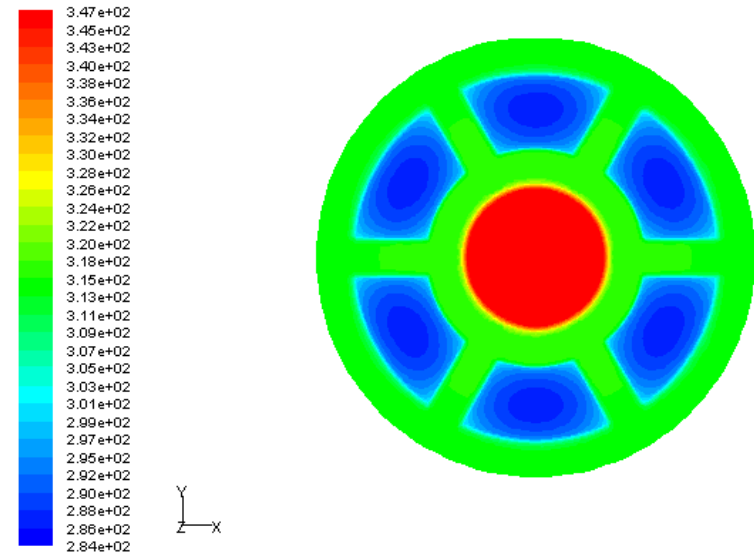

Figure 4. Temperature contours of liquid inlet and gas outlet and IHX walls (in Kelvin).

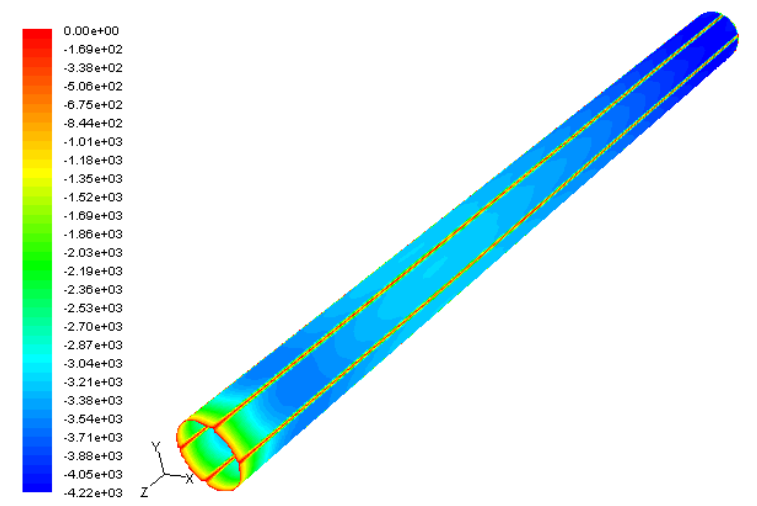

Figure 5. Heat transfer coefficient contours along the central channel (in $\mathrm{w} / \mathrm{m}^{2} \mathrm{~K}$ ).

\section{Acknowledgements}

The authors would like to thank Sardsaz Khodro co. for carrying out the experiments.

\section{References}

1. J. Navarro, R. Cabello, E. Torrela, Experimental evaluation of the internal heat exchanger influence on a vapor compression plant energy efficiency working with R22, R134a and R407C, Journal of energy, 30, 621-636 (2005)
2. S. Bahrami, H. Mohammadbeigi, M.H. Sabour, Experimental analysis of internal heat exchanger for automotive $\mathrm{A} / \mathrm{C}$ system, Proceeding of ASME conference, ESDA 2010, 24485 (2010)

3. Y. Chen, J. Gu, The optimum high pressure for $\mathrm{CO} 2$ transcritical refrigeration systems with internal heat exchangers, International Journal of Refrigeration, 28, 1238-1249 (2005)

4. C. Aprea, A. Maiorino: An experimental evaluation of the transcritical $\mathrm{CO} 2$ refrigerator performances using an internal heat exchanger. International Journal of Refrigeration, 31, 1006-1011 (2008)

5. Y.B. Tao, Y.L. He, Z.G. Wu, Experimental study on the performance of $\mathrm{CO} 2$ residential air-conditioning system with an internal heat exchanger, Energy Conversion and Management, 51, 64-70 (2010)

6. J. Rigola, N. Ablanque, C.D. Perez-Segarra, A. Olive, Numerical simulation and experimental validation of internal heat exchanger influence on $\mathrm{CO} 2$ transcritical cycle performance, International Journal of Refrigeration, 33, 664-674 (2010)

7. S.G. Kim, Y.J. Kim, G. Lee, M.S. Kim, The performance of a transcritical $\mathrm{CO} 2$ cycle with an internal heat exchanger for hot water heating, International Journal of Refrigeration, 28, 1064-1072 (2005)

8. H. Cho, C. Ryu, Y. Kim, Cooling performance of a variable speed $\mathrm{CO} 2$ cycle with an electronic expansion valve and internal heat exchanger, International Journal of Refrigeration, 30, 664-671 (2007)

9. R. Mastrullo, A.W. Mauro, S. Tino, G.P. Vanoli, A chart for predicting the possible advantage of adopting a suction/liquid heat exchanger in refrigerating system, Journal of Applied Thermal Engineering, 27, 2443-2448 (2007)

10. S.A. Klein, D.T. Reindl, K. Brownell, Refrigeration system performance using liquid-suction heat exchangers, International Journal of refrigeration, 23, 588-596 (2000) 